\title{
Problemas de Comportamento em Crianças com Transtorno Autista
}

\author{
Márcia Regina Fumagalli Marteleto ${ }^{1}$ \\ Teresa Helena Schoen-Ferreira \\ Brasília Maria Chiari \\ Jacy Perissinoto \\ Universidade Federal de São Paulo
}

\begin{abstract}
RESUMO - Este trabalho teve por objetivo identificar problemas de comportamento apresentados por crianças com Transtorno Autista. Participaram 118 mães de crianças de três a quinze anos, divididas em três grupos: crianças autistas; crianças com distúrbios de linguagem; e crianças sem patologias informadas. As mães responderam ao Child Behavior Checklist. Os grupos Transtorno Autista e Distúrbios de Linguagem tiveram escores médios significantemente maiores que o grupo de crianças sem patologias informadas. O grupo com Distúrbios de Linguagem teve escores médios maiores em comportamento agressivo e comportamentos externalizantes. As crianças com transtorno autista tiveram escores médios maiores em Problemas de Pensamento e menores em Ansiedade. Crianças com transtorno autista apresentam problemas de comportamento diferentes de crianças sem patologias informadas.
\end{abstract}

Palavras-chave: transtorno autista; problemas de comportamento; Child Behavior Checklist.

\section{Behavioral Problems of Children with Autism Disorders}

\begin{abstract}
The purpose of this study was the identification of behavioral problems in children with autism disorders. In total 118 mothers of children between three and fifteen years of age responded to the Child Behavior Checklist. Three groups of children were differentiated: children with autism, children with language disorders, and typical schoolchildren. The autism and language disorder groups had significantly higher average total scores than the typical schoolchildren. The language disorder group had higher average scores on aggressive behavior and externalizing behavior. The children with autism disorders had higher average scores on thought problems and lower scores on anxiety. Children with autism showed different behavioral problems in comparison with the two remaining groups of children.
\end{abstract}

Keywords: pervasive disorder; autism; behavior problems; Child Behavior Checklist.

O Transtorno Autista é uma severa e crônica anormalidade do desenvolvimento infantil, apresentando um quadro de prejuízo severo na interação social, na comunicação e atividade lúdica (APA, 2002; Rutter, 2005).

$\mathrm{O}$ autismo pode ser considerado uma síndrome comportamental com etiologias múltiplas podendo haver disfunções neurobiológicas, genéticas e déficits psicológicos como base para os déficits cognitivos encontrados nesta patologia (Gillberg, 1990, Baron-Cohen \& Bolton, 1993; Batley, Phillips e Rutter, 1996; Towbin, 1997, Rutter, 2005, Dworzynski, Happé, Bolton, \& Ronald, 2009, David \& cols., 2010, Spek, Scholte, \& van Berckelaer-Onnes, 2010).

A partir do momento que o autismo deixou de ser considerado psicose, passando a ser enquadrado dentro dos transtornos globais do desenvolvimento (APA, 2002), os autores que o estudaram procuraram delinear critérios diagnósticos através da observação e descrição clínica de casos, fornecendo características comportamentais e psicológicas observadas nas crianças. A partir dessas características comportamentais, foi possível classificar a gravidade, mensurar progressos ou retrocessos e programar intervenções e validá-las, desse modo foram elaborados diversos instrumentos de

1 Endereço para correspondência: Universidade Federal de São Paulo. Departamento de Distúrbios da Comunicação Humana. Rua Botucatu, 802 - Vila Clementino mensuração ou escalas de avaliação (Rutter, 2005, Matson, Nebel-Schwalm, Matson, 2006, Pereira, 2007, Reichow \& Volkmar, 2010).

Diante de uma situação tão complexa, a partir da observação e do diagnóstico destas crianças, verifica-se a necessidade da utilização de instrumentos para triagem de problemas de comportamento, pois possibilitam o rigor da observação e do registro dos comportamentos (Matson \& cols., 2006, Posserud, Lundervold, Lie, \& Gillberg, 2010).

Os comportamentos atípicos que caracterizam esta síndrome se manifestam de maneira heterogênea com diferentes níveis de gravidade. Podemos encontrar crianças que falam e outras que não falam; crianças sem nenhum tipo de contato social e outras com relacionamento atípico; além disto, algumas crianças podem apresentar retardo mental e outras quociente intelectual dentro da variação média normal (Lampreia, 2004, Bejerot, 2007). Esta heterogeneidade é que impulsiona o clínico em busca de instrumentos sensíveis e específicos para ajudar no diagnóstico diferencial e na identificação de diferentes problemas que as crianças com autismo podem apresentar (Matson \& cols., 2006; Williams \& Brayne, 2006).

Alguns comportamentos emitidos pela criança podem levar a família ou os profissionais, de modo especial o pediatra, a suspeitar de surdez. A criança com autismo, não atende pelo nome, não olha para o interlocutor, não segue o olhar; não olha para o chocalho quando este é balançado, apresenta 
atraso importante para fala. Quando querem alguma coisa se jogam no chão, se mordem, batem a cabeça, ou agridem e mordem o cuidador.

Outros comportamentos permitem uma confusão no diagnóstico com retardo mental (Bildt \& cols., 2004). A criança com autismo apresenta movimentos estereotipados, balança as mãos, corre de um lado para o outro, insiste em manter determinados objetos consigo, fixa somente numa característica do objeto, apresenta atraso no desenvolvimento da coordenação motora fina, grossa e de linguagem, demora para adquirir o controle esfincteriano e habilidades da vida diária, como comer com a colher, abotoar a camisa ou sentar. Também não apresenta autocuidado, como tomar banho sozinho, escovar os dentes, se proteger do fogo, atravessar a rua.

Os testes de triagem tanto podem ajudar no diagnóstico, como dificultá-lo ao introduzir comportamentos que são característicos de transtornos de desenvolvimento de diferente natureza. Os trabalhos realizados com os diversos instrumentos utilizam diferentes populações, tanto para aprimorar o instrumento quanto para afinar o olhar dos profissionais que lidam com a criança (Magyar \& Pandolfi, 2007, Rutgers, Van IJzendoorn, Bakermans-Kranenburg, \& Swinkels, 2007).

O Child Behavior Checklist - CBCL - é um dos instrumentos mais utilizados para fazer rastreamentos de comportamentos problemáticos (Achenbach, 1991). Trata-se de um questionário dividido em duas partes. A primeira parte se refere às competências sociais dos indivíduos. A segunda parte é composta por 113 itens, que identifica problemas de comportamento em crianças e adolescentes de 4 a 18 anos, a partir de informações fornecidas pelos pais. É um instrumento que se destaca pelo rigor metodológico com que foi elaborado, pelo seu valor na pesquisa e na prática clínica. As escalas do CBCL apresentam boa validade e confiabilidade e fornecem os sintomas psicopatológicos encontrados na infância e na adolescência.

O CBCL já foi traduzido em mais de 30 culturas diferentes e tem sido empregado internacionalmente em pesquisas epidemiológicas, diagnóstico, comparações entre culturas (Brown \& Achenbach, 1995, Ivanova \& cols., 2007, Rescorla \& cols., 2007).

Pesquisadores vêm desenvolvendo trabalhos com o CBCL procurando identificar suspeitas de autismo na população em geral e para apreender conjuntos de sintomas mais comuns em crianças autistas (Bölte, Dickhut \& Poustka, 1999, Duarte, 2001, Duarte, Bordin, Oliveira \& Bird, 2003).

O CBCL indicou diferentes padrões de ajustamento, onde crianças sem transtorno global do desenvolvimento apresentavam mais comportamentos externalizantes ou escores totais mais altos quanto comparados a crianças com autismo ou com algum outro transtorno global do desenvolvimento (DiSalvo \& Oswald, 2004). Bölte e cols. (1999) observaram que crianças com transtorno autista obtêm escores significativamente mais altos nas síndromes Problemas de Atenção, Problemas Sociais e Problemas com o Pensamento, medido pelo CBCL. Kuusikko e cols. (2008), entrevistando pais finlandeses de crianças com autismo ou Asperger, encontraram escores mais altos no CBCL em Comportamentos Internalizantes, Isolamento, Queixa Somática e Ansiedade/depressão, que o grupo controle.
Um estudo com a versão japonesa do CBCL observou que o teste identificava grandes dificuldades em comportamentos de comunicação e adaptação social por parte de jovens autistas, além de problemas de atenção e impulsividade (Kobayashi \& Murata, 1998).

Duarte e cols.(2003) examinaram a utilidade do CBCL/418 anos para identificar crianças com autismo na população em geral. Foram entrevistados 101 responsáveis, divididos em três grupos: 36 crianças com autismo, 31 com desordens psiquiátricas e 34 crianças em escola de ensino fundamental. A idade das crianças variou de 4 a 11 anos. Foi encontrado um agrupamento no CBCL que se denominou de Autístico/ Bizarro e o agrupamento denominado Problemas de Pensamento diferenciou as crianças autistas daquelas com outras desordens psiquiátricas e das escolares.

Limperopoulos e cols. (2008), após estudarem com o CBCL crianças nascidas pré-termo e com baixo peso, concluíram que o rastreio precoce de sinais de autismo pode ser justificado em populações de risco. Sikora e cols. (2008) também concluíram ser o CBCL um instrumento útil para análise comportamental e rastreamento de problemas de comportamento em crianças com autismo, demonstrando boa sensibilidade e especificidade.

O presente trabalho teve por objetivo verificar problemas de comportamento apresentados por crianças com transtorno autístico, distinguindo-os de crianças com transtorno de linguagem e de crianças escolares.

\section{Método}

Estudo transversal, observacional, aprovado pelo Comitê de Ética em Pesquisa da Unifesp, com a devida autorização dos serviços envolvidos, bem como assinatura do termo de consentimento livre e esclarecido.

\section{Participantes}

Foram convidadas para participar deste trabalho, mães de crianças com diagnósticos clínicos previamente estabelecidos por especialistas na área e também mães de crianças sem patologias informadas. Estas mães foram divididas em três grupos.

O primeiro grupo constituiu-se de $35(29,66 \%)$ mães de crianças com diagnóstico multiprofissional de Transtorno Autista estabelecido pelo DSM-IVTR (APA, 2002), com idade entre 3 e 15 anos, com idade média de 7 anos e 6 meses. A idade média de escolaridade materna foi de nove anos.

O segundo grupo constituiu-se de 31 (26,27\%) mães de crianças portadoras de transtornos de linguagem oral específica. Estas crianças tinham idade entre 3 e 11 anos, com idade média de 7 anos e 6 meses. A idade média de escolaridade materna foi de seis anos.

O terceiro grupo foi composto de $52(44,06 \%)$ mães de crianças sem patologias informadas, inseridas em Escola de Educação Infantil ou Ensino Fundamental. Neste grupo as crianças tinham idade entre 3 e 11 anos com idade média de 7 anos e 7 meses. A idade média de escolaridade materna foi de nove anos. 


\section{Instrumento}

O instrumento utilizado para este estudo foi a segunda parte do Child Behavior Checklist - CBCL (Achenbach, 1991), pois identifica os problemas de comportamento em crianças e adolescentes. Este instrumento foi traduzido e pré-adaptado por Bordin, Mari e Caieiro (1995). Esta seção do CBCL é composta por 113 itens referentes a problemas de comportamentos que a mãe deve avaliar dando um valor numérico de 0 , se o comportamento é ausente; 1 , se o comportamento é às vezes presente; ou 2 , se o comportamento é frequentemente presente.

O somatório dos escores obtidos permite ao avaliador traçar um perfil comportamental da criança ou adolescente, derivado da análise de oito agrupamentos de itens: I - Retraimento; II - Queixas Somáticas; III - Ansiedade/Depressão; IV - Problemas com o Relacionamento Social; V - Problemas com o Pensamento; - VI - Problemas com a Atenção; VII - Comportamento Delinquente; VIII - Comportamento Agressivo. O somatória dos três primeiros agrupamentos forma a Escala de Internalização e dos agrupamentos; VII e VIII formam a Escala de Externalização, detectadas e nomeadas desta forma a partir de várias análises multivariadas de problemas emocionais realizadas pelos autores do teste (Achenbach,1991). Nos agrupamentos, o escore para a categoria não clínica deve ser inferior a 67; para a categoria limítrofe ser de 67 a 70, inclusive; e, para a categoria clínica, ser maior que 70. Em relação às escalas, este índice deve ser inferior a 60 para a categoria não clínica; para a categoria limítrofe, ser de 60 a 63, inclusive; e, para a categoria clínica, ser maior que 63. Para pesquisas, o manual sugere dois agrupamentos: não clínico e clínico+limítrofe.

\section{Procedimento}

Após as mães terem assinado o termo de consentimento livre e esclarecido, o CBCL foi aplicado na forma de entrevista conduzida pelo aplicador para facilitar a compreensão das questões. Foi pedido para as mães pensarem e responderem sobre o comportamento atual dos seus filhos. As mães dos grupos um e dois foram entrevistadas enquanto seus filhos estavam em atendimento especializado, e as mães do grupo três, enquanto seus filhos estavam em sala de aula.

O CBCL foi digitado diretamente no programa de computador específico, utilizando os escores norte-americanos. Desta forma foram obtidos os escores por escalas e por agrupamentos. Foram calculadas as médias e desvios-padrão de cada agrupamento e escala do CBCL por grupo.

\section{Análise de Dados}

Os resultados são apresentados através de medidas descritivas para observar as variáveis. Foi utilizado o teste de Kruskal-Wallis para comparar os três grupos em todas as variáveis quantitativas. Caso observada diferença, utilizou-se o teste de Mann-Whitney para especificar onde de fato ela ocorreu. Foi considerado o nível de significância $\mathrm{p} \leq 0,05$.

\section{Resultados}

A Tabela 1 apresenta as médias e os desvios-padrão encontrados nesta amostra. Observa-se que, no escore Total de Problemas e Comportamento Internalizante, a média de todos os grupos esteve inserida na categoria Clínica, e na escala Comportamento Externalizante, a média do grupo autista não foi classificada como clínica. Estes resultados foram analisados utilizando o teste Kruskal-Wallis.

A média do grupo Transtorno Autista pode ser classificada na categoria clínica em três agrupamentos e a do grupo distúrbios de linguagem em uma categoria. Observa-se na Tabela 2 quais agrupamentos ou escalas estiveram associadas aos grupos. Somente nos agrupamentos Comportamento Delinquente e $\mathrm{T}$ externalizante não existiu diferença significante entre os grupos.

Utilizando o teste Mann-Witney podemos observar que as crianças do grupo Transtorno Autista e Distúrbios de Linguagem tiveram escores médios $\mathrm{T}$ total significantemente maiores que o grupo de escolares típicos. O grupo de crianças com Distúrbios de Linguagem teve escores médios maiores no comportamento agressivo e nos comportamentos externalizantes do que o grupo Transtorno Autista.

As crianças com Transtorno Autista tiveram escores médios maiores na categoria problemas de pensamento. $\mathrm{Na}$ categoria ansiedade, o escore médio foi significantemente menor no grupo de crianças com Transtorno Autista do que nos outros dois grupos.

As crianças inseridas em escola de educação infantil e ensino fundamental demonstraram menor tendência ao isolamento, a problemas sociais e aos problemas de atenção que os outros dois grupos.

\section{Discussão}

Utilizar escores de outra população é uma dificuldade para o pesquisador brasileiro. Apesar de no Brasil já haverem vários estudos utilizando o CBCL, como os de Figueiredo e Schermann (2001), Costa e Silvares (2003), Saud e Tonelotto (2005) ou Gutt (2005), são utilizados os escores americanos. Acreditamos que, por estarmos com três grupos homogêneos quanto à idade e à escolaridade materna, minimizamos o efeito que poderia advir de se utilizar os escores americanos.

Estudos de validação em outros países (Nho, 2000; Roussos \& cols., 2001) vêm mostrando que há uma tendência de se obter escores maiores que os americanos. Talvez essa seja a razão de ter sido encontrado tantos escores médios na categoria clínica. Faz-se necessário a validação de instrumentos para sua melhor utilização no Brasil.

O grupo de escolares obteve escores médios menores em todas as escalas, com exceção das escalas de ansiedade e de agressividade, onde o grupo de autistas teve os menores escores. Apesar das crianças com Transtorno Autístico terem poucas habilidades sociais, a agressividade não é uma característica (APA, 2002). Os estereótipos que estas crianças costumam apresentar podem até assustar as pessoas, entretanto, as mães percebem que seus filhos não são agressivos. Estes comportamentos - como não permitir ser tocado, ficar batendo a cabeça na parede, ficar se balançando - não tem 
Tabela 1. Médias, desvios-padrão e teste Kruskal-Wallis em cada categoria do CBCL segundo os grupos.

\begin{tabular}{|c|c|c|c|c|c|}
\hline Categorias & Grupos & $n$ & $M$ & $D P$ & $\chi^{2}$ \\
\hline \multirow{3}{*}{ Isolamento } & Autista & 35 & 67,03 & 11,85 & \\
\hline & Dist. Ling. & 31 & 65,06 & 11,26 & $18,58 * * *$ \\
\hline & Escolares & 52 & 57,48 & 7,66 & \\
\hline \multirow{3}{*}{ Queixa somática } & Autista & 35 & 56,00 & 7,22 & \\
\hline & Dist. Ling. & 31 & 61,58 & 9,47 & $6,87^{*}$ \\
\hline & Escolares & 52 & 57,90 & 7,49 & \\
\hline \multirow{3}{*}{ Ansiedade/depressão } & Autista & 35 & 59,80 & 6,48 & \\
\hline & Dist. Ling. & 31 & 66,61 & 10,43 & $8,66^{*}$ \\
\hline & Escolares & 52 & 62,65 & 9,52 & \\
\hline \multirow{3}{*}{ Problemas sociais } & Autista & 35 & 64,97 & 9,87 & \\
\hline & Dist. Ling. & 31 & 64,10 & 10,35 & $16,46^{* * *}$ \\
\hline & Escolares & 52 & 57,21 & 8,86 & \\
\hline \multirow{3}{*}{$\begin{array}{l}\text { Problemas com o pensa- } \\
\text { mento }\end{array}$} & Autista & 35 & 71,89 & 8,42 & \\
\hline & Dist. Ling. & 31 & 63,19 & 10,30 & $41,59 * * *$ \\
\hline & Escolares & 52 & 56,90 & 7,14 & \\
\hline \multirow{3}{*}{ Problemas de atenção } & Autista & 35 & 72,03 & 11,28 & \\
\hline & Dist. Ling. & 31 & 69,45 & 12,80 & $30,74^{* * *}$ \\
\hline & Escolares & 52 & 59,17 & 8,33 & \\
\hline \multirow{3}{*}{$\begin{array}{l}\text { Comportamento delin- } \\
\text { quente }\end{array}$} & Autista & 35 & 56,69 & 7,35 & \\
\hline & Dist. Ling. & 31 & 59,26 & 9,77 & 1,81 \\
\hline & Escolares & 52 & 55,98 & 6,92 & \\
\hline \multirow{3}{*}{ Comportamento agressivo } & Autista & 35 & 59,14 & 9,25 & \\
\hline & Dist. Ling. & 31 & 66,00 & 12,71 & $6,07^{*}$ \\
\hline & Escolares & 52 & 60,13 & 9,12 & \\
\hline \multirow{3}{*}{$\mathrm{T}$ total de problemas } & Autista & 35 & 67,43 & 7,62 & \\
\hline & Dist. Ling. & 31 & 68,84 & 9,69 & $17,64 * * *$ \\
\hline & Escolares & 52 & 60,42 & 10,19 & \\
\hline \multirow{3}{*}{$\mathrm{T}$ internalizante } & Autista & 35 & 63,69 & 7,84 & \\
\hline & Dist. Ling. & 31 & 67,32 & 10,14 & $8,57^{*}$ \\
\hline & Escolares & 52 & 60,79 & 9,90 & \\
\hline \multirow{3}{*}{$\mathrm{T}$ externalizante } & Autista & 35 & 57,74 & 9,36 & \\
\hline & Dist. Ling. & 31 & 63,39 & 11,37 & 5,30 \\
\hline & Escolares & 52 & 58,00 & 9,59 & \\
\hline
\end{tabular}

$* \mathrm{p}<0,05 ; * * \mathrm{p}<0,01 ; * * * \mathrm{p}<0,001$. 
Tabela 2. Comparações das médias entre os grupos por agrupamento e escala

\begin{tabular}{|c|c|c|c|}
\hline Grupos & & $\begin{array}{c}\text { Autista } \\
\text { U }\end{array}$ & $\begin{array}{c}\text { Dist. Ling. } \\
\mathrm{U}\end{array}$ \\
\hline \multirow{2}{*}{ Isolamento } & Dist. Ling. & 488,5 & - \\
\hline & Escolares & $462,5^{* * *}$ & $473,0 * *$ \\
\hline \multirow{2}{*}{ Queixa somática } & Dist. Ling. & $352,0^{*}$ & - \\
\hline & Escolares & 765,5 & 626,5 \\
\hline \multirow{2}{*}{ Ansiedade/depressão } & Dist. Ling. & $315,0 * *$ & - \\
\hline & Escolares & 765,0 & 600,5 \\
\hline \multirow{2}{*}{ Problemas sociais } & Dist. Ling. & 516,5 & - \\
\hline & Escolares & $500,5 * * *$ & $474,5^{*}$ \\
\hline \multirow{2}{*}{ Problemas com o pensamento } & Dist. Ling. & $282,0 * * *$ & - \\
\hline & Escolares & $183,0 * * *$ & $519,5^{* *}$ \\
\hline \multirow{2}{*}{ Problemas de atenção } & Dist. Ling. & 450,5 & - \\
\hline & Escolares & $327,0 * * *$ & $386,0 * * *$ \\
\hline \multirow{2}{*}{ Comportamento delinquente } & Dist. Ling. & ns & - \\
\hline & Escolares & ns & ns \\
\hline \multirow{2}{*}{ Comportamento agressivo } & Dist. Ling. & $370,0^{*}$ & - \\
\hline & Escolares & 833,0 & $589,0^{*}$ \\
\hline \multirow{2}{*}{$\mathrm{T}$ total de problemas } & Dist. Ling. & 500,5 & - \\
\hline & Escolares & $510,5^{* *}$ & $434,5^{* * *}$ \\
\hline \multirow{2}{*}{$\mathrm{T}$ internalizante } & Dist. Ling. & 398,0 & - \\
\hline & Escolares & 772,5 & $507,0 * *$ \\
\hline \multirow{2}{*}{ T externalizante } & Dist. Ling. & ns & - \\
\hline & Escolares & ns & ns \\
\hline
\end{tabular}

$* \mathrm{p}<0,05 ; * * \mathrm{p}<0,01 ; * * * \mathrm{p}<0,001$

por objetivo ferir as outras pessoas, mas proteger a si mesmo de algo que não conseguem lidar (Rutgers \& cols., 2007).

As crianças com problemas de linguagem obtiveram escores mais altos neste agrupamento. Resultado semelhante encontrado no estudo de DiSalvo e Oswald (2004). A falta de habilidades linguísticas para se comunicar pode ter levado este grupo de crianças a desenvolver comportamentos problemáticos - neste caso específico - para mostrar o que desejam. As mães dessas crianças observam que seus filhos invadem o espaço dos outros, possivelmente na tentativa de mostrar seu próprio espaço (Reichow, \& Volkmar, 2010; Spek \& cols., 2010).

A linguagem funciona como um regulador interno, facilitando à assunção de comportamentos adequados ao fim proposto. Este grupo de crianças, na percepção de suas mães, falham ao encontrar um comportamento que surta o mesmo efeito da conduta problemática, mas que seja socialmente adequado, concordando com as afirmações de David e cols. (2010).

Além do comportamento agressivo, as mães dessas crianças com problemas de linguagem identificaram seus filhos como tendo mais problemas externalizantes (comportamentos voltados contra outras pessoas ou coisas) e mais comportamentos delinquentes do que as crianças com transtorno autista e que as crianças escolares. Crianças com estas características acabam tendo muita dificuldade para aceitar limitações im- postas pelo ambiente familiar e social. E as mães também têm muita dificuldade em orientar seus filhos a buscarem outras soluções para resolver os conflitos que cotidianamente advém à vida das crianças, como afirma Zuñiga (2005).

A linguagem permite a busca de soluções alternativas, especialmente na negociação. Essas crianças precisam desenvolver habilidades para selecionar os melhores comportamentos que facilite o convívio social e a manifestação de seus sentimentos e desejos. É interessante que para uma melhor prática do fonoaudiólogo, este se utilize também de instrumentos que rastreiam problemas de comportamento (Zuñiga, 2005).

As crianças do grupo autístico foram identificadas por suas mães como tendo mais comportamentos do tipo Problemas com o Pensamento e Problemas de Atenção do que as crianças do grupo transtorno de linguagem ou do grupo de escolares. Estes resultados são semelhantes aos encontrados por Kobayashi e Murata (1998) e Bölte, Dickhut e Poustka (1999). Trabalhos em diferentes culturas vêm confirmando a presença desses comportamentos atípicos em indivíduos com transtorno autista. São agrupamentos que vão ao encontro das características que o DSM IV ${ }^{\mathrm{TR}}$ (APA, 2002) e a CID 10 (OMS, 1993) colocam para o Transtorno Autístico.

As mães observam que seus filhos ficam perdidos em seus próprios pensamentos, como se estivessem em outro mundo, o qual é difícil para elas entrarem ou compreenderem. Essas 
características afetam sobremaneira a dinâmica familiar, pois exigem que os responsáveis pela criança autista desenvolvam algumas habilidades específicas para compreender os desejos e necessidades da criança e poder responder a contento, já que apresenta dificuldades em ajustar seu comportamento ao contexto social.

O isolamento é uma forma que estes indivíduos parecem desenvolver para conviverem melhor com suas dificuldades. Neste estudo, apesar das crianças com transtorno autista obterem escores mais altos que os dois outros grupos, não houve diferença estatística entre eles quanto ao agrupamento isolamento (os escolares é que se diferenciaram dos dois outros grupos). As mães de crianças com transtorno autista afirmam que as crianças gostam de estar com elas e com outras pessoas próximas, mas preferem não ter intimidade com indivíduos com os quais têm pouco contato. Como o questionário é respondido pelas mães, precisamos considerar que no nosso país o contato físico afetivo é uma forma cultural de manifestação de sentimentos e de comportamentos, o que pode minimizar a identificação do comportamento de isolamento no caso do transtorno autista. A amizade com pessoas fora do âmbito familiar nem sempre é valorizada, podendo ser substituída por algum tipo de relacionamento com a família extensa. A proximidade afetiva que a criança autista deste estudo apresenta com os pais ou outros parentes próximos, mesmo que restrita, funciona como facilitador para o convívio social. Não entendemos, por parte da mãe que preencheu o instrumento, como uma negação dos comportamentos do filho, mas os pais se adaptam e descobrem facetas amáveis de seu filho, centrando-se em maneiras de ajudá-los a enfrentar os problemas, como sugerido por Reichow e Volkmar (2010).

As crianças do grupo autista apresentaram escores significativamente mais baixos no agrupamento Ansiedade/Depressão. Acreditamos que tal resultado se deva às características da síndrome, que dificulta a identificação de comportamentos do tipo ansioso ou depressivo. Roer as unhas, por exemplo, pode ser identificado como comportamento estereotipado do autista, e não como manifestação de ansiedade especificamente. É necessário analisar e descobrir o propósito do comportamento problemático. Em crianças com transtorno do desenvolvimento, é bem mais difícil descobrir a intenção da conduta, o que, de forma alguma, elimina a necessidade de encontrá-la e ajudar a criança a se expressar de forma menos destrutiva, concordando com David e cols. (2010).

Os resultados sugerem que necessitam desenvolver habilidades sociais que facilitem uma inserção social mais bem sucedida e que a utilização de testes que rastreiem problemas de comportamento pode ajudar os profissionais que lidam com crianças com transtorno autista a avaliar as áreas que, no momento, mais necessitam ser trabalhadas. Os pais são os que mais conhecem a criança e, portanto, estão em melhor situação para valorar suas dificuldades e progressos.

As mães responderam ao teste em forma de entrevista. Em relação às mães do grupo autista, observou-se que foi um momento para que falassem com calma sobre seu filho e os sentimentos, preocupações e frustrações que advém de ser mãe de uma criança que necessita de apoio profissional para se desenvolver. Os serviços que prestam atendimento a este grupo de crianças sentem a necessidade de proporcionar oportunidades para que se fortaleça o sistema familiar, em especial as mães, e com isto possam ajudar seu filho a progredir.

O Child Behavior Checklist apontou diferenças nos escores entre crianças com transtorno autista, crianças com Distúrbios de Linguagem e crianças escolares típicas. Ao se analisar o resultado por agrupamentos, tal instrumento indicou que as crianças com transtorno autístico apresentam problemas de comportamento em consonância com as características da síndrome listadas no DSM IV ${ }^{\mathrm{TR}}$ ou na CID 10. Portanto, é uma ferramenta útil para triagem de crianças.

As mães de crianças com Transtorno Autístico perceberam seus filhos com problemas de comportamento diferentes de crianças típicas e daquelas com transtorno de linguagem oral específica.

Comportamentos relacionados a Problemas com o Pensamento e Problemas de Atenção estiveram relacionados ao grupo de crianças com autismo. Este grupo apresentou escores significativamente mais baixos de comportamentos indicativos de Ansiedade/Depressão e Agressividade.

\section{Referências}

Achenbach, T. M. (1991). Manual for the child behavior checklist/4-18 and 1991 profile. Burlington, VT: University of Vermont.

American Psychiatric Association (2002). DSM-IV-R, Diagnostic and statistical manual of mental disorders (4 $4^{\mathrm{a}}$ ed. revisada). Washington: APA.

Baron Cohen S., \& Bolton, P. (1993). Autism the facts. Oxford: Oxford University Press.

Batley, A., Phillips, W., \& Rutter, M. (1996). Autism towards an integration of clinical, genetic and neuropsychological, and neurobiological perspectives. Journal of Child Psychology and Psychiatry, 37, 89-126.

Bejerot, S. (2007). An autistic dimension: a proposed subtype of obsessive-compulsive disorder. Autism, 11, 101-110.

Bildt, A., Sytema, S., Ketelaars, C., Kraijer, D., Mulder, E., Volkmar, F., \& Minderaa, R. (2004). Interrelationship between autism diagnostic observation schedule-generic (ADOS-G), autism diagnostic interview-revised (ADI-R), and the diagnostic and statistical manual of mental disorders (DSM-IV-TR) classification in children and adolescents with mental retardation. Journal of Autism \& Developmental Disorders, 34, 129-137.

Bölte, S., Dickhut, H., \& Poustka, F. (1999). Patterns of parentreported problems indicative in autism. Psychopathology, 32, 93-97.

Bordin, I. A. S., Mari, J. J., \& Caeiro, M. F. (1995). Validação da versão brasileira do"Child Behavior Checklist" (CBCL) Inventário de Comportamentos da Infância e da Adolescência: dados preliminares. ABPAPAL, 17(2), 55-66.

Brown, J. S., \& Achenbach, T. M. (1995). Bibliography of published studies using the Child Behavior Checklist and related materials: 1995 edition. Burlington, VT: University of Vermont.

Costa, N. J. D., \& Silvares, E. F. M. (2003). Enurese na adolescência: estudo de caso com intervenção comportamental. Interação em Psicologia, 7(1), 9-17. 
David, N., Aumann, C., Bewernick, B. H., Santos, N. S., Lehnhardt, F. G. \& Vogeley, K. (2010). Investigation of mentalizing and visuospatial perspective taking for self and other in Asperger Syndrome. Journal of Autism \& Developmental Disorders, 40, 290-299.

DiSalvo, C. A., \& Oswald, D. P. (2004). Differential diagnosis of children with pervasive developmental disorders: The use of both autism-specific and general measures. Autism and related developmental disabilities, 20, 1-8.

Duarte, C. S. (2001). Características de personalidade de mães de crianças com diagnóstico de autismo infantil: um estudo comparativo. Tese de doutorado, Universidade Federal de São Paulo, São Paulo.

Duarte, C. S., Bordin, I. A. S., Oliveira, A., \& Bird, H. (2003). The CBCL and the identification of children with autism and related conditions in Brazil: Pilot findings. Journal of Autism and Developmental Disorders, 33(6), 703-707.

Dworzynski, K., Happé, F., Bolton, P., \& Ronald, A. (2009). Relationship between symptom domains in autism spectrum disorders: A population based twin study. Journal of Autism \& Developmental Disorders, 39, 1197-1210.

Figueiredo, C. K. B., \& Schermann, L. (2001). Interação mãecriança e problemas de comportamento infantil em crianças com hipotireoidismo congênito. Psicologia: Reflexão e Crítica, 14(3), 487-495.

Gillberg, C. (1990). Autism and pervasive developmental disorders. Journal of Child Psychology and Psychiatry, 31, 99-119.

Gutt, E. K. (2005). Perfil comportamental e competência social de crianças e adolescentes filhos de mulheres com esquizofrenia. Dissertação de Mestrado, Universidade de São Paulo, São Paulo.

Ivanova, M.Y., Dobrean, A., Dopfner, M., Erol, N., Fombonne, E., Fonseca, A.C., Frigerio, A., Grietens, H., Hannesdottir, H., Kanbayashi, Y., Lambert, M., Achenbach, T.M., Larsson, B., Leung, P., Liu, X., Minaei, A., Mulatu, M.S., Novik, T.S., Oh, K.J., Roussos, A., Sawyer, M., Simsek, Z., Dumenci, L., Steinhausen, H.C., Metzke, C.W. Wolanczyk, T., Yang, H. J., Zilber, N., Zukauskiene, R., Verhulst, F.C., Rescorla, L.A., Almqvist, F., Weintraub, S., Bilenberg, N., Bird, H., \& Chen, W. J. (2007). Testing the 8-syndrome structure of the CBCL in 30 societies. Journal of Clinical Child and Adolescent Psychology, 36, 405-417.

Kobayashi, R., \& Murata, T. (1998). Behavioral Characteristics of 187 young adults with autism. Psychiatry and Clinical Neurosciences, 52, 383-391.

Kuusikko, S., Pollock-Wurman, R., Jussila, K., Carter, A. S., Mattila, M. L., Ebeling, H., Pauls, D. L., \& Moilanen, I. (2008). Social anxiety in high-functioning children and adolescents with autism and Asperger Syndrome. Journal of Autism and Developmental Disorders, 38, 1697-1709.

Lampreia, C. (2004). Os enfoques cognitivista e desenvolvimentista no autismo: Uma análise preliminar. Psicologia: Reflexão e Crítica, 17, 111-120.

Limperopoulos, C., Bassan, H., Sullivan, N. R., Soul, J. S., Robertson, R. L. Jr., Moore, M., Ringer, A. S., Volpe J. J., $\&$ du Plessis, A. J. (2008). Positive screening for autism in ex-preterm infants: Prevalence and risk factors. Pediatrics, 121(4), 758-765.
Magyar, C. I., \& Pandolfi, V. (2007). Factor structure evaluation of the childhood autism rating scale. Journal of Autism and Developmental Disorder, 37, 1781 - 1794.

Matson, J. L., Nebel-Schwalm, M., \& Matson, M. L. (2006). A review of methodological issues in the differential diagnosis of autism spectrum disorders in children. Research in Autism spectrum Disorders, 1, 38-54.

Nho, C.R. (2000). Psychological well-being of Korean American and immigrant adolescents. Doctoral Dissertation, Columbia University, New York.

Organização Mundial da Saúde (1993). Classificação de transtornos mentais e de comportamento da CID 10: Descrições clínicas e diretrizes diagnósticas. Porto Alegre: Artes Médicas.

Pereira, A. M. (2007). Autismo Infantil: Tradução e validação da CARS (Childhood Autism Rating Scale) para uso no Brasil. Dissertação de Mestrado, Universidade Federal do Rio Grande do Sul, Porto Alegre.

Posserud, M., Lundervold, A. J., Lie, S. A., \& Gillberg, C. (2010). The prevalence of autism spectrum disorders: Impact of diagnostic instrument and non-response bias. Social Psychiatry and Psychiatric Epidemiology, 45, 319-327

Reichow, B., \& Volkmar, F. R. (2010). Social skills interventions for individuals with Autism: Evaluation for evidence-based practices within a best evidence synthesis framework. Journal of Autism \& Developmental Disorders, 40, 149-166.

Rescorla, L. A., Achenbach, T. M., Ivanova, M. Y., Dumenci, L., Almqvist, F., Bilenberg, N., Bird, H., Chen, W., Dobrean, A., Döpfner, M., Erol, N., Fombonne, E., Fonseca, A., Frigerio, A., Grietens, H., Hannesdottir, H., Kanbayashi, Y., Lambert,M., Larson, B., Leung, P., Liu, X., Minaei, A., Mulatu, M. S., Novik, T. S., Oh, K. J., Roussos, A., Sawyer, M., Simsek, Z., Steinhausen, H. C., Weintraub, S., Weisz, J., Metzke, C. W., Wolanczyk,T., Yang, H. J., Nelly Zilber, N., \& Zukauskiene, R. (2007). Problems reported by parents of children aged 6 to 16 in 31 cultures. Journal of Emotional and Behavioral Disorders, 15, 130-142.

Roussos, A., Francis, K., Zoubou, V., Kiprianos, S., Prokopiou, A., \& Richardson, C. (2001). The standardization of Achenbach's Youth Self-Report in Greece in a national sample of high school students. European Child \& Adolescent Psychiatry, 10(1), 47-53.

Rutgers, A. H., Van IJzendoorn, M. H., Bakermans-Kranenburg, M. J., \& Swinkels, S. H. N. (2007). Autism and attachment: The attachment Q-Sort. Autism, 11, 187-200.

Rutter, M. (2005). Aetiology of autism: findings and questions. Journal of Intellectual Disability Research, 49, 231-235.

Saud, L. F., \& Tonelotto, J. M. F. (2005). Comportamento social na escola: Diferenças entre gêneros e séries. Psicologia Escolar e Educacional, 9, 47-57.

Sikora, D. M., Hall, T. A., Hartley, S. L., Gerrard-Morris, A. E., \& Cagle, S. (2008). Does parent report of behavior differ across ADOS-G classifications: Analysis of scores from the CBCL and GARS. Journal of Autism and Developmental Disorders, 38(3), 440-448.

Spek, A., Scholte, E. M., \& van Berckelaer-Onnes, I. A. (2010). Theory of mind in adults with HFA and Asperger Syndrome. Journal of Autism \& Developmental Disorders, 40, 280-289.

Towbin, K. E. (1997). Autism and Asperger syndrome. Current Opinions in Pediatry, 9(4), 361-366. 
Williams, J., \& Brayne, C. (2006). Screening for autism spectrum disorders: What is the evidence? Autism, 10, 11-35.

Zuñiga, A. F. (2005). Descrição e tratamento dos transtornos da comunicação e da linguagem. Em V. E. Caballo \& M. A. Simon, (Orgs.). Manual de Psicologia Clínica Infantil e do

Recebido em 17.03.2009 Adolescente: Transtornos Especificos. Santos: Livraria Santos.

Primeira decisão editorial em 10.02.2010

Versão final em 11.03.2010

Aceito em 10.05.2010

\section{INVESTIGACIÓN E INNOVACIÓN PARA LA IGUALDAD: desafíos para la Psicología en América Latina}

Local: Montevideo, Uruguay

Período: 14 a 25 de fevereiro de 2011

III CONGRESO PSICOLOGÍA Y RESPONSABILIDAD SOCIAL: ABORDAJES INTERDISCIPLINARIOS

Local: Bogotá, Colômbia

Período: 07 a 09 de Abril 2011 